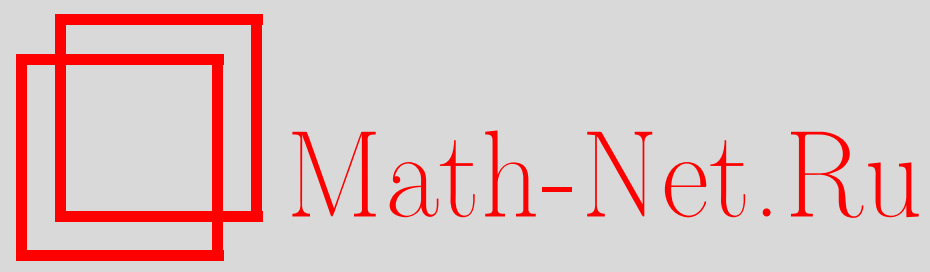

В. М. Галкин, Н. В. Мохнина, О некоторых автоморфизмах на ортогональных группах в нечетной характеристике, Матем. заметки, 2001, том 70, выпуск 1, 27-37

DOI: https://doi.org/10.4213/mzm715

Использование Общероссийского математического портала Math-Net.Ru подразумевает, что вы прочитали и согласны с пользовательским соглашением http://www.mathnet.ru/rus/agreement

Параметры загрузки:

IP : 18.207 .199 .55

26 апреля 2023 г., 13:52:08

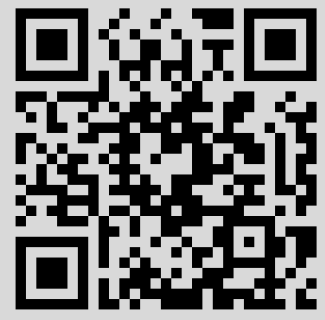




\title{
О НЕКОТОРЫХ АВТОМОРФИЗМАХ НА ОРТОГОНАЛЬНЫХ ГРУППАХ В НЕЧЕТНОЙ ХАРАКТЕРИСТИКЕ
}

\author{
В. М. Галкин, Н. В. Мохнина
}

В статье доказьвается, что простые ортогональные группы $O_{2 n+1}(q), O_{2 n}^{ \pm}(q)(q$ нечетно) не могут быть группами автоморфизмов конечных леводистрибутивных квазигрупп. Это частньй случай гипотезы, по которой группа автоморфизмов леводистрибутивной квазигруппы разрешима. Для полного доказательства гипотезы необходимо проверить все простые группы.

Библиография: 11 названий.

1. В статье доказьвается отсутствие на простых ортогональных группах $O_{2 n+1}(q)$, $O_{2 n}^{ \pm}(q)$ ( $q$ нечетно) нетождественного автоморфизма $\varphi$ со свойством

$$
x \varphi\left(x^{-1}\right) \in s T s^{-1} \Longrightarrow x \in T,
$$

где $T$ - подгруппа $\varphi$-неподвижных элементов.

Это очередной шаг в реализации программы доказательства гипотезы о разрешимости групп трансляций $Z(G)$ конечных леводистрибутивных квазигрупп $G(\cdot)$, сводящейся к $\varphi$-гипотезе: проверке отсутствия нетождественного автоморфизма $\varphi$ с указанньп свойством у конечной простой группы П. Группа левых трансляций $Z(G)$ по определению порождена левыми трансляциями $L_{a}=(x \rightarrow a \cdot x)$. Группы, для которых существует нетривиальньй автоморфизм $\varphi$, назьваются далее $\varphi$-группами. Сведения о них можно найти в [1]-[3]. Отметим следующие из свойств: $\varphi$-структура наследуется при переходе к $\varphi$-инвариантным подгруппам и факторгруппам по $\varphi$-инвариантным нормальным делителям; на смежных классах $\Pi / T$, где П - рассматриваемая простая группа, можно ввести структуру леводистрибутивной квазигрупш, если положить $x \cdot y=x \varphi\left(x^{-1} y\right)$. Каждая квазигруппа может быть получена таким образом (хотя и с неоднозначно определенной группой П). Однако каждое представление можно редуцировать к минимальному, уже однозначно определенному квазигрупшой. Для этого от П переходим к главной подгрупе $\Pi_{1}=\left\langle x \varphi\left(x^{-1}\right), x \in \Pi\right\rangle$, порожденной каноническими элементами, $\mathrm{a}$ затем профакторизуем по наибольшему нормальному делителю, содержащемуся в $T$. Группа П для минимального представления есть коммутант группы левых трансляций квазигруппы $G$.

Работа выполнена при финансовой поддержке Российского фонда фундаментальных исследований, грант № 99-01-00398. 
ПРЕДЛОЖЕНИЕ 1. Пусть П - конечная $\varphi$-групnа, $T \subset \Pi-$ nодгрупnа $\varphi$-неподвижных әлементов. Индекс $\left(\Pi: \Pi_{1}\right)$ делит $|T|-$ порядок группы $T$.

ДокАЗАТЕЛЬСТво. Представления П/T и $\Pi_{1} / \Pi_{1} \cap T$ приводят к одной и той же квазигруппе. Следовательно, $(\Pi: T)=\left(\Pi_{1}: \Pi_{1} \cap T\right)$, откуда $\left(\Pi: \Pi_{1}\right)=\left(\Pi_{1}: \Pi_{1} \cap T\right)$, что делит $|T|$.

2. Описание ортогональных квазигрупп и их автоморфизмов можно найти в [4]-[6]. Ниже приводятся необходимые для дальнейшего изложения сведения.

Группа $O_{2 n+1}(q)$. Пусть $(x, y)$ - невырожденная билинейная симметрическая форма на $(2 n+1)$-мерном пространстве $V$ над конечным полем $F_{q}$ из $q$ элементов. В последовательности групा $\Gamma O_{2 n+1} \supset G_{2 n+1} \supset S O_{2 n+1} \supset \Omega_{2 n+1}=O_{2 n+1} \Gamma O_{2 n+1}$ группа полулинейньх преобразований [5], $G O_{2 n+1}$ - подгруппа изометрий формы $(x, y)$, $S O_{2 n+1}$ - подгруппа преобразований с определителем $1, \Omega_{2 n+1}=O_{2 n+1}$ - коммутант группы $S O_{2 n+1}$. Автоморфизмы группы $O_{2 n+1}$ в классическом представлении определяются как сопряжение элементами из Г $O_{2 n+1}$. Группа $O_{2 n+1}$ проста при $n \geqslant 2$. Имеют место следующие изоморфизмы: $O_{3}(q) \cong L_{2}(q), O_{5}(q) \cong S p_{4}(q)$.

Групша $O_{2 n}^{ \pm}(q)$. В аналогичной последовательности групп $\Gamma O_{2 n}^{ \pm} \supset G O_{2 n}^{ \pm} \supset S O_{2 n}^{ \pm} \supset$ $\Omega_{2 n}^{ \pm} \supset Z\left(\Omega_{2 n}^{ \pm}\right)$обе группы $O_{2 n}^{ \pm}$возникают как факторгруппы $\Omega_{2 n}^{ \pm} / Z\left(\Omega_{2 n}^{ \pm}\right)$по центру $Z\left(\Omega_{2 n}^{ \pm}\right)=1$ или $Z_{2}$, соответствующиевыбору двух видов неэквивалентных билинейных форм $(x, y)$. Автоморфизмы групш $O_{2 n}^{-}$определяются так же, как и в группе $O_{2 n+1}$. При $n>4$ автоморфизмы групш $O_{2 n}^{+}$вновь индуцируются сопряжениями элементами из $Г O_{2 n}^{+}$. При $n=4$ ситуация усложняется в связи с появлением так назьваемой “тройственности". Группа $O_{2 n}^{+}$является простой при $n \geqslant 3$, группа $O_{2 n}^{-}-$при $n \geqslant 2$; $O_{4}^{+}(q) \cong L_{2}(q) \times L_{2}(q), O_{4}^{-}(q) \cong L_{2}\left(q^{2}\right), O_{6}^{+}(q) \cong L_{4}(q), O_{6}^{-}(q) \cong U_{4}(q)$.

Ортогональные групшы $O_{2 n+1}, O_{2 n}^{+}, O_{2 n}^{-}$можно рассматривать как групш Шевалле типов $B_{n}, D_{n},{ }^{2} D_{n}$. Они порождаются корневыми группами $\left\langle x_{\alpha}(t)\right\rangle$, где $\alpha \in \Sigma-$ система корней в схеме Дьнкина, $t \in F_{q}, x_{\alpha}(t+s)=x_{\alpha}(t) x_{\alpha}(s)$. Для наших целей необходимо выделить в ортогональных группах наличие картановской подгруппы $H=\left\langle h_{\alpha}(t) \mid t \in F_{q}^{*}\right\rangle$, образующие которой определенным образом выражаются через элементы корневых подгрупп, причем $h_{-\alpha}(s)=h_{\alpha}\left(s^{-1}\right), h_{\alpha}(t+s)=h_{\alpha}(t) h_{\alpha}(s)$. Группа $H$ абелева, а факторгруппа $N(H) / H=W$ может быть отождествлена с группой Вейля системы корней $\Sigma$, т.е. группой изометрий $\Sigma$, порожденной отражениями $r_{\alpha}(u \rightarrow u-2((u, \alpha) /(\alpha, \alpha)) * \alpha)$. При этом $\omega_{\alpha} h_{\beta}(t) \omega_{\alpha}^{-1}=h_{\omega(\beta)}(t)$ для $\omega \in W$.

Автоморфизмы ортогональных групп классифицируются стандартным образом, если использовать интерпретацию групп как групп Шевалле [6]. Каждьй автоморфизм есть композиция полевого, диагонального, внутреннего и графового автоморфизмов. Такое деление автоморфизмов относительно. Оно зависит от выбора базиса в пространстве $V$.

В группах Шевалле различают унипотентные и полупростые элементы. Под последними понимаются элементы, чьи порядки взаимно просты с характеристикой поля $q$.

Лемма 1. В группах $O_{2 n+1}(q), O_{4 m}^{ \pm}(q)$ ( $q$ нечетно) полупростой әлемент нечетного порядка сопряжен со своим обратным. 
ДокАЗАТЕЛЬСТво. Соответствующие алгебраические групшы будем рассматривать как группы Шевалле. Сопряжение элементов с помощю элемента $\omega \in W$ из группы Вейля дает $\omega h_{\alpha}(t) \omega^{-1}=h_{\omega(\alpha)}(t)$, где $h_{\alpha}(t), h_{\omega(\alpha)}(t)$ - элементы картановской подгрупшы $H$. Из соотношения $h_{\alpha}(t) x_{\beta}(u) h_{\alpha}\left(t^{-1}\right)=x_{\beta}\left(t^{\langle\beta, \alpha\rangle} u\right)$ [6] следует $h_{-\alpha}(t)=h_{\alpha}\left(t^{-1}\right)$, по крайней мере, с точностью до множителя из центра групш. Существует элемент $\omega \in W$ такой, что $\omega(\alpha)=-\alpha$ при $\forall \alpha$. Таковым является произведение отражений относительно $n$ ортогональных корней, которые в рассматриваемых группах легко выделяются из системы корней $\Sigma$. Полупростой элемент можно вложить в картановскую подгрупу. Если $x=\prod_{\alpha} h_{\alpha}\left(t_{\alpha}\right)$, то, используя $h_{-\alpha}(t)=h_{\alpha}\left(t^{-1}\right)$, получаем

$$
\omega x \omega^{-1}=\prod_{\alpha} h_{\omega(\alpha)}\left(t_{\alpha}\right)=\prod_{\alpha} h_{-\alpha}\left(t_{\alpha}\right)=\prod_{\alpha} h_{\alpha}\left(t_{\alpha}^{-1}\right)=x^{-1},
$$

т.е. $x \sim x^{-1}$ в алгебраической групе. Поскольку фундаментальная группа, соответствующая алгебраической групп, есть $Z_{2}$ (или $Z_{2} \times Z_{2}$ ), то сопряженность элемента со своим обратньм имеет место и в группах $O_{2 n+1}(q), O_{4 m}^{ \pm}(q)[7]$.

3. В этом пункте рассматривается $\varphi$-гипотеза для групшы $O_{2 n+1}$. В рассуждении используется индуктивное предположение, что $\varphi$-структура тривиальна для ортогональных групп с размерностью, меньшей $2 n+1$, а для группы $O_{2 n+1}$ предполагаем, что она является нетривиальной $\varphi$-группой. В наименьшем контрпримере можно считать $n \geqslant 3$ из-за описанных вьше изоморфизмов. Доказательство $\varphi$-гипотезы можно разбить на следуюшие этапы.

3.1. Поскольку известные простые групшы не имеют регулярных автоморфизмов [4], то автоморфизм $\varphi$ нерегулярен. Тогда $T$ либо состоит только из унипотентных элементов, либо содержит полупростые элементы.

3.2. Докажем, что $T$ содержит полупростые элементы. Допустим противное. Без ограничения общности можно считать, что $T \subseteq U=\left\langle x_{\alpha}(t), \alpha>0\right\rangle-\varphi$-инвариантная $p$-подгруппа $(p / q)$.

Нормализатор $N(T)$ $\varphi$-инвариантен, $T \subset N(T)$. Индуцированное действие $\varphi$ на $N(T) / T$ регулярно. Значит, можно выделить $\varphi$-инвариантную $p$-подгруппу в $N(T) / T$ [8]. Прообраз ее в $N(T)$ дает $\varphi$-инвариантную $p$-подгруппу $\Gamma$, строго содержащую $T$, если $T \neq U ; N(\Gamma) \varphi$-инвариантен. Продолжая рассуждения, устанавливаем существование $\varphi$-инвариантной силовской $p$-подгруппы, в качестве которой берем $U$.

Нормализатор $N(U)$-инвариантен. Фактор $N(U) / U$ изоморфен картановской подгруппе $H$, т.е. на $H$ д действует регулярно.

С другой стороны, в $H$ есть подгруппа $H_{0}=\left\langle h_{\alpha}(t), t \in F_{p}\right\rangle\left(t \neq 1, F_{p}\right.$ - простое подполе), элементы которой выдерживают полевую, диагональную, внутреннюю и графовую часть автоморфизма $\varphi$, т.е. в $H$ есть $\varphi$-неподвижные элементы. Противоречие. Следовательно, $T$ не может состоять только из унипотентных элементов.

3.3. На этом этапе докажем, что подгрупша $T$ содержит инволюцию.

Возьмем полупростой элемент $t \in T$. Групша, порожденная элементом $t$, имеет $\varphi$-инвариантньй нормализатор $N\langle t\rangle$. Фактор $N\langle t\rangle /\langle t\rangle$ приводит к некоторой квазигруппе $G$. 
Для $x \in N\langle t\rangle$, используя лемму 1 , имеем $x t x^{-1}=t^{-1}$ и $\varphi(x) t \varphi\left(x^{-1}\right)=t^{-1}$, т.е. $x^{-1} \varphi(x) \in C(t)$, где $C(t)$ - централизатор элемента $t$. Подгруппа

$$
N_{1}(C(t))=N_{1}\langle t\rangle=\left\langle x \varphi\left(x^{-1}\right), x \in N\langle t\rangle\right\rangle \subset C(t) \neq N\langle t\rangle .
$$

В дальнейшем эти рассуждения будем назьвать $C-N$-конструкиией.

Согласно предложению 1 в $|T|$ входит множитель $\left(N\langle t\rangle: N_{1}\langle t\rangle\right)$. Он четен, так как четен уже $(N\langle t\rangle: C(t))$. В самом деле, в $N\langle t\rangle / C(t)$ есть элемент $u: u t u^{-1}=t^{-1}$; повторное сопряжение дает $u^{2} t u^{-2}=u t^{-1} u^{-1}=\left(u t u^{-1}\right)^{-1}=t$, т.е. $u^{2} \in C(t)$, но $u \notin C(t)$. Значит, в $N\langle t\rangle / C(t)$, а следовательно, и в $T$ есть инволюция $u$.

3.4. Рассмотрим инволюцию $\sigma \in T$. В группе $O_{2 n+1}$ в нечетной характеристике $\sigma^{2}=1$ и векторное пространство $V$ разлагается в прямую сумму $V^{+} \oplus V^{-}, V^{+} \perp V^{-}$, $\operatorname{dim} V^{-}$четно; $\forall x \in V^{+}: \sigma(x)=x ; \forall x \in V^{-}: \sigma(x)=-x$.

Известно, что $\varphi(x)=u x u^{-1}$ при некотором $u \in \Gamma O_{2 n+1}$. Пусть $C_{G O}(\sigma)$ - централизатор инволюции в соответствующей групе $G O_{2 n+1}$.

ПРЕДЛОЖЕНИЕ 2. $u \in C_{G O}(\sigma)$ переводит в себя $V^{ \pm}$.

ДоказАТЕЛьСтво. Так как $\sigma-\varphi$-неподвижный элемент, то $u \sigma u^{-1}=\sigma$.

Возьмем $x \in V^{+}$. Имеем $\sigma u(x)=u \sigma(x)=u(x) \in V^{+}$. Если $x \in V^{-}$, то $\sigma u(x)=$ $u \sigma(x)=-u(x) \in V^{-}$.

Централизатор $\sigma$ в групе $G O_{2 n+1}$ разлагается в произведение $\varphi$-инвариантных множителей, $C_{G O}(\sigma)=G O\left(V^{+}\right) \times G O\left(V^{-}\right)$. Поскольку $n \geqslant 3$, то либо $\operatorname{dim} V^{+}$, либо $\operatorname{dim} V^{-}$не менее 4. Пусть для определенности $\operatorname{dim} V^{+} \geqslant 4$. Тогда факторгруппа $\Omega\left(V^{+}\right) / Z\left(\Omega\left(V^{+}\right)\right)$проста при $\operatorname{dim} V^{+}<4$ и полупроста при $\operatorname{dim} V^{+}=4$, так как $\Omega_{4}\left(V^{+}\right) / Z\left(\Omega_{4}\left(V^{+}\right)\right) \cong O_{4}^{+}(q) \cong L_{2}(q) \times L_{2}(q)$. При $q=3$ группа $L_{2}(q)$ разрешима, поэтому группу $O_{7}(3)$ как исключение рассмотрим ниже.

Действие автоморфизма $\varphi$ определено следующим образом: $\varphi(x)=u x u^{-1}, u \in$ $\Gamma O_{2 n+1}$. Групша $\Omega\left(V^{+}\right)$-тривиальна по индукции, так как тривиально действие $\varphi$ на $\Omega\left(V^{+}\right) / Z\left(\Omega\left(V^{+}\right)\right)$и на $Z\left(\Omega\left(V^{+}\right)\right)$. Следовательно, $u-$ скаляр на $V^{+}$.

В пространстве $V^{+}$выделим подпространство $W$ коразмерности 1 , на котором метрика невырождена, и рассмотрим ортогональную группу на пространстве $W \oplus V^{-}$. Eе можно рассматривать как $\varphi$-инвариантную подгруппу в ортогональной групе на $V$, так как $u(W)=W$. На ней $\varphi=1$ по индукции, т.е. $u-$ скаляр на $W \oplus V^{-}$, а потому $u-$ скаляр на $V$, т.е. $\varphi=1$ на групше $O_{2 n+1}$.

Рассмотрим автоморфизм $\varphi$ на группе $O_{7}(3)$.

Так как полевая часть у $\varphi$ отсутствует, то $\varphi(x)=u x u^{-1}$, где $u \in S O_{7}(3)$ является диагональным автоморфизмом. Как известно, квадрат диагонального автоморфизма внутренний, т.е. $\varphi^{2}(x)=u^{2} x u^{-2}$, где $u^{2} \in O_{7}(3)$. Элемент $u^{2}$ не содержит полупростой части. В противном случае из-за сопряженности полупростой части $u_{1}^{2}$ с обратной следует $C\left(u_{1}^{2}\right) \neq N\left\langle u_{1}^{2}\right\rangle$. По $C$ - $N$-конструкции получаем противоречие. Поэтому $u^{2}-$ унипотент. Некоторая степень его $v=u^{2 \alpha}$ - элемент порядка 3. Согласно атласу [9] $v \sim v^{-1}$. Далее, $C-N$-конструкция, примененная к $C(v)$, дает противоречие.

4. В этом пункте докажем, что подгруппа $T$ групшы $O_{4 m+2}^{ \pm}(q)$ содержит инволюцию.

Пусть это не так. Возьмем в $T$ элемент $x$ наименьшего порядка $t$. Очевидно, что $t$ простое нечетное. Элемент $x$ может быть либо унипотентом, либо полупростым. 


\section{1. Пусть $x$ полупрост, т.е. $(t, q)=1$.}

Лемма 2. Если $x$ - әлемент простого порядка $t$, то $x$ сопряэен $c x^{q}$ в групne $O_{4 m+2}^{+}(q)$, a в әpynne $O_{4 m+2}^{-}(q)-c x^{q^{2}}$.

ДокАЗАтЕльСтво. Группу $G=O_{4 m+2}^{+}(q)$ вложим в алгебраическую групу $\bar{G}$, определенную над алгебраическим замьканием $\bar{F}_{q}$ поля $F_{q}$. Полупростой элемент $x$ лежит в некотором максимальном торе. Ввиду того, что все максимальные торы сопряжены, можно считать, что $x$ лежит в картановской подгруппе группы $\bar{G}$. Автоморфизм Фробениуса оставляет $x$ инвариантным и возводит в степень $q$ при переходе $x$ в $y=s x s^{-1}(s \in \bar{G})$ из картановской подгруппы, т.е. $x \sim x^{q}$ в группе $\bar{G}$. А так как порядок $t$ нечетен, то сопряженность элементов в $\bar{G}$ влечет их сопряженность и в $G$ [7].

Для групшы $O_{4 m+2}^{-}(q)$ теорема доказывается аналогично. Отличие составляет основное поле $F_{q^{2}}$.

Лемма 3. В Т есть либо әлемент простого порядка $t \mid q-1$ в группе $O_{4 m+2}^{+}(q)$ и $t \mid q^{2}-1$ в группе $O_{4 m+2}^{-}(q)$, либо унипотентный әлемент.

ДоКАЗАТЕЛЬСТВО проведем для группы $O_{4 m+2}^{+}(q)$.

Предположим противное. Пусть для $x$ из $T\left(x^{t}=1\right.$, где $t$ наименьшее) $t \nmid q$ и $t \nmid q-1$. Тогда $t$ должно делить некоторое число $q^{i}-1(i>1)$. (В групше $O_{4 m+2}^{-}(q) t$ должно делить $q^{i}-(-1)^{i}, i>2$.) Ясно, что $x \neq x^{q}$ и $C(x) \neq N\langle x\rangle$. Значит, $i /(N\langle x\rangle: C(x))$ есть делитель $t-1$. Противоречие с выбором $x$.

В доказательстве для группы $O_{4 m+2}^{-}(q) q$ заменяется на $q^{2}$.

Итак, $x$ - полупростой элемент в $T$ порядка $t \mid q-1$ в группе $O_{4 m+2}^{+}(q)$ и $t \mid q^{2}-1$ в группе $O_{4 m+2}^{-}(q)$. Многочлен $f(x)=x^{t}-1$ разложим на неприводимые в $F_{q}$ множители $f_{i}(x)$. Тогда $V=\Sigma \oplus V_{i}$, где $V_{i}$ аннулируется многочленом $f_{i}(x)$. Каждое слагаемое $V_{i}$ можно рассматривать как пространство над полем $F_{q}(x)$. Распределим слагаемые $V_{i}$ на самосопряженные $V_{j}$ и пары сопряженных друг с другом $V_{k} \sim V_{k^{\prime}}$.

Слагаемое $V_{j}$ будем назьвать самосопряжсенным, если оно аннулируется не только $f_{j}(x)$, но и $f_{j}\left(x^{-1}\right)$. Тогда $V=\Sigma \oplus V_{j} \oplus \Sigma \oplus\left(V_{k}+V_{k^{\prime}}\right)$. Ограничение формы на $V_{i}$ невырождено. Доказательство не приводится, но понятие об этом можно найти в п. 5 .

Рассмотрим структуру централизатора полупростого элемента $x$.

Если $t \mid q-1$, то $x$ - картановский элемент с собственными значениями $1, \ldots, 1, \lambda_{1}$, $\lambda_{1}^{-1}, \ldots$, где $\lambda_{i} \neq 1 ; x^{-1}$ имеет те же собственные значения, поэтому $x \sim x^{-1}$ в группе $G O_{4 m+2}^{ \pm}(q)$ с помощью ортогонального преобразования $u$. При наличии единиц среди собственных значений $x$ в централизатор $\widehat{C}(x)$ входит множитель $G O\left(V_{j}\right)$. Тогда в $\widehat{C}_{G O}(x)$ входят элементы $G O \backslash S O, S O \backslash \Omega$. Если $u \in \Omega$, то $x \sim x^{-1}$ в $\Omega$. Если $u \in S O \backslash \Omega$, то $u$ заменим на $u_{1}=u c \in \Omega$, где $c \in S O \backslash \Omega$, и $x \sim x^{-1}$ в группе $\Omega_{4 m+2}$ с помощью ортогонального преобразования $u_{1}$. Если $u \in G O \backslash S O$, то $u$ заменим на $u_{2}=u c_{1} \in S O$, где $c_{1} \in G O \backslash S O$. Таким образом, $x \sim x^{-1}$ в группе $\Omega_{4 m+2}$ и по $C-N$-конструкиии в $T$ имеем инволюцию.

Если среди собственных значений $x$ нет единиц, но, по крайней мере, одно из $V_{k}$ неодномерно, то в централизаторе $\widehat{C}_{G O}(x)$ появляется множитель $G L\left(V_{k}\right)$. Он неразрешим при $V_{k}>2$, что приводит к четности порядка $T$.

Если $t \nmid q-1$, а $t \mid q+1$ (в группе $\left.O_{4 m+2}^{-}(q)\right)$, то $\Pi f_{i}(x)$ имеет квадратичные множители и множитель $x-1$. Если $\exists V_{j}$, где $x \equiv 1$, то проходят прежние рассуждения. 
Если среди собственных значений нет единиц, то $\widehat{C}_{G O}(x)$ будет содержать нормальным делителем группу $U_{n}(q)$, а она $\varphi$-тривиальна [10], т.е. двойка делит порядок $T$.

Если среди собственных значений $x$ нет единиц и все $V_{i}$ одномерны при $t \mid q-1$ (двумерны при $\left.t \mid q^{2}-1\right)$, то справедливы следующие рассуждения. $\widehat{C}(x)$ есть $\varphi$-инвариантная подгрупша диагональных матриц, т.е. картановская. Нормализатор $N(\widehat{C}(x)) \varphi$-инвариантен. Фактор $N(\widehat{C}(x)) / \widehat{C}(x)-\varphi$-группа, являющаяся группой Вейля $W$, изоморфной симметрической группе $\Sigma_{n}$. Знакопеременная групп $A_{n}$ характеристична, поэтому фактор $\Sigma_{n} / A_{n}=Z_{2}$ - тривиальная $\varphi$-группа, так как не существует квазигрупп порядка 2 [1]. Отсюда 2 делит $|T|$. (В случае $t \mid q^{2}-1$ групша Вейля есть типа $B_{n-1}=\Sigma_{2 m} \cdot 2^{m}[6]$, но это не меняет суть рассуждений.)

Итак, если в $T$ элемент наименьшего порядка полупрост, то это инволюция.

4.2. Пусть теперь элемент $x$ наименьшего порядка $t$ является унипотентом в $T$.

Базис в пространстве $V$ можно выбрать таким образом, чтобы оно разлагалось в прямую сумму подпространств двух типов: $e$-пространства и $\left(f, f^{\prime}\right)$-пространства [7]. Базис $e$-пространства представляют следующие векторы: $e, s e, s^{2} e, \ldots, s^{k-1} e$, где $s^{k}=0$, $k$ нечетно. На нем задана метрика: $(e, e)=0,\left(e, s^{k-1} e\right)=\omega,\left(e, s^{2} e\right)=\left(e, s^{4} e\right)=\cdots$ $=\left(e, s^{k-3} e\right)=0$ ( $\omega$ определяется с точностью до квадратного множителя). Остальные произведения определяются однозначно; $\left(f, f^{\prime}\right)$-пространство имеет размерность $2 k$ ( $k$ четно). Базис $f, s f, s^{2} f, \ldots, s^{k-1} f, f^{\prime}, s f^{\prime}, s^{2} f^{\prime}, \ldots, s^{k-1} f^{\prime}\left(s^{k} f=s^{k} f^{\prime}=0\right)$ с метрикой

$$
\left(f, s^{i} f\right)=\left(f^{\prime}, s^{i} f^{\prime}\right)=0, \quad\left(f, s^{i} f^{\prime}\right)= \begin{cases}0, & i>k-1 \\ 1, & i=k-1\end{cases}
$$

ЛЕмма 4. Если $x$ - унипотент, то $x \sim x^{m^{2}}$ в группе $O_{4 m+2}^{ \pm}(q)$.

ДокАЗАТЕЛьство. Так как унипотент $x$ можно представить в виде $x=1+s, s^{k}=0$, то $x^{m}=1+T$, где $T=m s+C_{m}^{2} s^{2}+\cdots+s^{m}$. На $\left(e, f, f^{\prime}\right)$-пространстве из-за единственности нормализации базиса $x \sim x^{m}$ с помощью ортогонального преобразования $u: s^{i} e \rightarrow T^{i} e_{1}, s^{i} f \rightarrow T^{i} f_{1}, s^{i} f^{\prime} \rightarrow T^{i} f_{1}^{\prime}$, где $T e_{1}, T f_{1}, T f_{1}^{\prime}$ - базисные векторы для $x^{m}\left(u \in G O_{4 m+2}^{ \pm}(q)\right)$. Значения их скалярных произведений такие же, как для векторов $s^{i} e, s^{i} f, s^{i} f^{\prime}$. Если $u \in S O_{4 m+2}^{ \pm}(q)$, то $u^{2} \in O_{4 m+2}^{ \pm}(q)$. Откуда $x \sim x^{m^{2}}$ в группе $O_{4 m+2}^{ \pm}(q)$.

Так как пространство $V$ есть прямая сумма $e$ - и $\left(f, f^{\prime}\right)$-подпространств, с одной стороны, и имеет размерность $4 m+2$, с другой, то в $V$ обязательно должно быть $e$-подпространство нечетной размерности. Поскольку $e \in C(x)$, то и $(-e) \in C(x)$, но $|e|=(-1)^{k}=-1$. Значит, в $C(x)$ есть элемент из $G O \backslash S O$. Если $u \in G O \backslash S O$, то $u$ заменим на $u_{1}=u c$, где $c \in G O \backslash S O$. Тогда $x$ будет сопряжен с $x^{m}$ с помошью элемента $u_{1} \in S O$, т.е. $x \sim x^{m^{2}}$ в групе $O_{4 m+2}^{ \pm}(q)$.

Если $x \neq x^{m^{2}}$, то $C(x) \neq N\langle x\rangle$ и в $T$ найдутся элементы менъшего порядка, чем $t$. Противоречие. Поэтому $x=x^{m^{2}}$. Возьмем $m=2$. Тогда найдется $x$ такое, что $x^{3}=1$, и $q$ - степень тройки.

Рассмотрим централизатор унипотентного элемента $x$ :

$$
\widehat{C}_{G O}(x)=R\left(\prod_{i \text { чет. }} \widehat{S} p_{2 r_{i}}(q) \times \prod_{i \text { нечет. }} G O_{r_{i}}(q)\right),
$$


где $R$ - унипотентньй радикал, т.е. наибольший нормальньй делитель, состоящий из унипотентных элементов

$$
|R|=q^{\operatorname{dim} R}, \quad \operatorname{dim} R=\frac{1}{2} \sum_{i}\left(r_{i}+r_{i+1}+\cdots\right)^{2}-\sum_{i} r_{i}^{2}-\frac{1}{2} \sum_{i \text { чет. }} r_{i}
$$

где $r_{i}$ - число подпространств размерности $i$ при $i$ нечетном и размерности $2 i$ при $i$ четном [7].

Исключение составляют случаи: $r_{1}, r_{3} \leqslant 4 ; r_{2} \leqslant 1$ в поле характеристики $q=3$. Наибольшая при этом размерность векторного пространства $V$ составляет $18(\operatorname{dim} V=$ $\left.1 r_{1}+2 r_{2}+3 r_{3}=18\right) ; e$-подпространство выбираем следующим образом: $e_{1}, e_{2}, e_{3}, e_{4}$. Унипотент $x$ тождественен на $e$-подпространстве $(k=1, s=0)$. Так как $e$-подпространство обязательно есть в $V$, то всегда можно выбрать такой базис. Тогда в $\widehat{C}_{G O}(x)$ входят элементы $G O \backslash S O, S O \backslash \Omega$. Как отмечалось выше, $x \sim x^{m}$ в группе $G O_{4 m+2} \mathrm{c}$ помощью ортогонального преобразования $u$. Возьмем $m=-1$. Если $u \in \Omega$, то $x \sim x^{-1}$ в $\Omega$. Если $u \in S O \backslash \Omega$, то $u$ заменим на $u_{1}=u c \in \Omega$, где $c \in S O \backslash \Omega$, и $x \sim x^{-1}$ в группе $\Omega_{4 m+2}$ с помощью ортогонального преобразования $u_{1}$. Если $u \in G O \backslash S O$, то $u$ заменим на $u_{2}=u c_{1} \in S O$, где $c_{1} \in G O \backslash S O$. Таким образом, при $r_{1}, r_{3} \leqslant 4$ $x \sim x^{-1}$ в групше $\Omega_{4 m+2}$. Следовательно, $C$ - $N$-конструкция опять приводит к наличию в $T$ инволюции.

5. Докажем $\varphi$-гипотезу для груп $O_{2 n}^{ \pm}(q)$. (Групша $O_{8}^{+}(q)$ рассматривается здесь без наличия в ней тройственности.) Доказательство проведем методом от противного. В наименьшем контрпримере берет $n \geqslant 4$. В доказательстве используем индуктивное предположение о верности $\varphi$-гипотезы для ортогональных групш размерности меньшей $2 n$.

Рассмотрим инволюцию $\sigma \in T$. (Наличие в $T$ инволюции для групшы $O_{4 n}^{ \pm}(q)$ описано в п. 3 при рассмотрении группы $O_{2 n+1}(q)$, для группы $O_{4 n+2}^{ \pm}(q)$ - в п. 4.)

При $Z(\Omega)=1$ доказательство $\varphi$-тривиальности группы $O_{2 n}^{ \pm}$аналогично доказательству $\varphi$-тривиальности групшы $O_{2 n+1}$.

При $Z(\Omega)=2$ в нечетной характеристике два типа инволюций: $\sigma^{2}=1$ - инволюция I типа и $\sigma^{2}=-1$ - инволюция II типа. В случае инволюции I типа рассуждения о $\varphi$-тривиальности аналогичны описанньм в п. 4.3. Инволюции II типа существуют, когда $-1 \in \Omega$. Для этого необходимо и достаточно, чтобы $\operatorname{spin}(-1)=(-1)^{n} \omega^{ \pm}$являлся квадратом, где $\omega^{ \pm}-$квадрат и неквадрат в поле $F_{q}$ соответственно [11]. При $q \equiv 1(\bmod 4)$ используется $\omega^{+}$, т.е. инволюция II рода может быть лишш в $O_{2 n}^{+}(q)$. Еcли же $q \equiv-1(\bmod 4)$, то ее существование в $O_{2 n}^{+}(q)$ возможно лиш при четном $n$, а при $n$ нечетном - только в $O_{2 n}^{-}(q)$.

5.1. $\sigma^{2}=-1, q \equiv 1(\bmod 4)$, т.е. $-1=\lambda^{2}$, где $\lambda \in F_{q}$.

Векторное пространство $V$ разлагается в прямую сумму $V^{+} \oplus V^{-}$. При этом $\forall x \in V^{+}$: $\sigma(x)=\lambda x ; \forall x \in V^{-}: \sigma(x)=-\lambda x$.

ПРЕДЛОЖЕНИЕ 3. $V^{ \pm}$самоортогональньl, $\operatorname{dim} V^{+}=\operatorname{dim} V^{-}$. 
ДокАЗАТЕльство. Для определенности возьмем $x, y \in V^{+}$и подействуем на них $\sigma$.

Имеем $(x, y)=(\sigma(x), \sigma(y))=\lambda^{2}(x, y)=-(x, y)$. Это возможно лишь при $(x, y)=0$, т.е. $V^{+} \perp V^{+}\left(V^{-} \perp V^{-}\right.$доказьвается аналогично). Из самоортогональности $V^{ \pm}$ следует сопряженность $V^{+}$и $V^{-}$. В противном случае метрика на $V^{+} \oplus V^{-}$будет вырождена. Таким образом, $\operatorname{dim} V^{+}=\operatorname{dim} V^{-}$.

Выясним структуру централизатора инволюции.

Выберем базис $e_{1}, e_{2}, \ldots, e_{n} \in V^{+}$:

$$
e_{1}^{\prime}, e_{2}^{\prime}, \ldots, e_{n}^{\prime} \in V^{-}, \quad\left(e_{i}, e_{i}^{\prime}\right)=1, \quad\left(e_{i}, e_{j}^{\prime}\right)=0 \text { при } i \neq j .
$$

Выбор осуществляется путем выделения в пространстве $V$ гиперболических плоскостей. При этом пространство $V$ разлагается в виде их прямой суммы [11].

Элемент $u \in \widehat{C}_{G O}(\sigma)$ такой, что $u \sigma u^{-1}=\sigma$, переводит в себя $V^{ \pm}$. (Доказательство аналогично доказательству предложения 2.)

Действие $u$ на $V^{+}$можно задать произвольно матрицей $u_{i j} \in G L_{n}(q)$. Тогда действие $u$ на $V^{-}$определим при помощи матрицы $u_{j i} \in G L_{n}(q): u\left(e_{i}^{\prime}\right)=u_{j i}^{\prime} e_{j}$. Можно показать, что $\left(e_{i}, e_{j}^{\prime}\right)=\delta_{i j}=u_{j i}^{*} u_{j i}^{\prime}$, где $u_{j i}^{*}$ - матрица, транспонированная к $u_{j i}$. Таким образом, $u_{j i}^{\prime}=\left(u_{j i}^{*}\right)^{-1}$ и элемент $u \in \widehat{C}_{G O}(\sigma)$ представляется матрицей

$$
\left(\begin{array}{ll}
u_{i j} & \\
& u_{i j}^{*}-1
\end{array}\right)
$$

Следовательно, $\widehat{C}_{G O}(\sigma) \cong G L_{n}(q)=S L_{n} \times Z_{q-1}=L_{n} \times Z\left(L_{n}\right) \times Z_{q-1}$. Заметим, что определители элементов централизатора $|u|=1$ и $\widehat{C}_{G O}(\sigma)=C_{S O}(\sigma)$.

ЗАмЕчаниЕ 1 . Накрытие $\widehat{C}_{G O}(\sigma)$ состоит из элементов $u \in G O_{2 n}^{ \pm}(q)$, которые коммутируют с $\sigma$ точностью до знака: $u \sigma u^{-1}= \pm \sigma$.

$\widehat{C}_{G O}(\sigma)$ выдерживает автоморфизм $\varphi$, поскольку $\widehat{C}_{G O}(\sigma)$ получается из простого сечения $L_{n}(n \geqslant 4)$ расширением с помощью $\varphi$-тривиальных групш $Z\left(L_{n}\right)$ и $Z_{q-1}$. Так как имеет место последовательность включений $\widehat{C}_{G O} \supset C_{G O} \supseteq C_{S O} \supseteq C_{\Omega} \supset Z_{2}$, то $C_{\Omega}(\sigma)$ будет также $\varphi$-тривиален.

Мызнаем, что $\varphi(x)=u x u^{-1}$ при некотором $u \in \Gamma O_{2 n}^{ \pm}(q)$. Так как $\sigma-\varphi$-неподвижньй элемент, то $u \sigma u^{-1}= \pm \sigma$. Инволюция $\sigma$ такая, что $u \sigma u^{-1}=-\sigma$ под действием $\varphi$ меняет местами блоки $u_{j i}$ и $\left(u_{j i}^{*}\right)^{-1}$ и, значит, автоморфизм $\varphi$ не может быть тривиальньм на сечении $L_{n}(q)$. Поэтому $u \sigma u^{-1}=\sigma$.

По индукции $u$ - скаляр на $V^{ \pm}$. Выделим в пространстве $V$ подпространство $W$ коразмерности 2 такое, при котором $V=W \oplus\left\langle e_{1}, e_{1}^{\prime}\right\rangle$, и рассмотрим ортогональную группу на $W$. По индуктивному предположению группа $O_{2 n-2}^{ \pm} \varphi$-тривиальна, т.е. $u$ - скаляр и при том один и тот же на обоих блоках. Значит, $u= \pm 1$ и $\varphi=1$ на группе $O_{2 n}^{ \pm}$. Противоречие.

5.2. $\sigma^{2}=-1, q \equiv-1(\bmod 4), V^{+} \perp V^{-}$и $V^{ \pm}$самосопряжены. Здесь ортогональная форма “поднимается" до унитарной $(x, y)=0.5 \operatorname{Tr}\{x, y\}$, где $\{x, y\}$ - невырожденная эрмитова форма и след берется над $F_{q^{2}} / F_{q}$. В этом случае

$$
\widehat{C}_{G O}(\sigma) \cong G U_{n}(q)=S U_{n} \times Z_{q+1}=U_{n} \times Z\left(U_{n}\right) \times Z_{q+1}
$$


Можно показать, что $\widehat{C}_{G O}(\sigma)=C_{S O}(\sigma), \widehat{C}_{G O}(\sigma)-\varphi$-тривиально.

Полулинейное преобразование можно продолжить и на унитарную группу, поскольку эрмитова форма выражается через ортогональную $\{x, y\}=(x, y)-\sigma(\sigma x, y)$.

Как и прежде, $\varphi(x)=u x u^{-1}$ при некотором $u \in \Gamma O_{2 n}^{ \pm}(q)$. Выделим в пространстве $V$ подпространство $W$ меньшей размерности и рассмотрим на нем ортогональную подгруппу исследуемой групшы. По индуктивному предположению она $\varphi$-тривиальна, т.е. $u$ - скаляр на $W$, а значит, и на $V$. Поэтому $\varphi=1$ на групе $O_{2 n}^{ \pm}$. Противоречие.

Исключение из общей схемы рассуждения составляет группа $O_{8}^{+}(3)$, так как структура централизатора ее инволюции I типа не содержит простой части (состоит из произведения множителей вида $\left.L_{2}(3)\right)$. Как говорилось вьше, автоморфизм $\varphi$ есть сопряжение элементом $u \in \Gamma O_{8}^{+}(3)$, но тогда $u^{2} \in G O_{8}^{+}(3)$. Нечетная часть $\left(u^{2}\right)^{\prime}$ может быть только 3-элементом $\left(\left(u^{2}\right)^{\prime} \in \Omega_{8}^{+}(3)\right)$. Согласно атласу [9] 3-элемент сопряжен со своим обратньм, что запрещает $C$ - $N$-конструкция. Поэтому $u^{2}-2$-элемент, а значит, порядок $\varphi$ - это степень двойки и $T$ содержит силовскую 2 -подгруппу. Но тогда $T$ содержит инволюции всех типов, а инволюции II типа исключения не составляют, так как в структуру их централизаторов входят группы $U_{n}(q)$ и $L_{n}(q)$, которые при $n \geqslant 4$ просты.

6. Докажем $\varphi$-гипотезу для групшы $O_{8}^{+}(q)$ при наличии в ней графового автоморфизма третьего порядка (автоморфизма “тройственности").

При рассмотрении централизатора $\widehat{C}_{G O}(\sigma)$ инволюции, содержащейся в $T$, вьше отмечалось, что при инволюции I типа $\widehat{C}_{G O}(\sigma)=C_{G O}(\sigma) \cong G O_{6}^{ \pm}(q) \times G O_{2}^{ \pm}(q)$ или $\widehat{C}_{G O}(\sigma)=C_{G O}(\sigma) \cong G O_{4}^{ \pm}(q) \times G O_{4}^{ \pm}(q) ;$ при инволюции II типа $\widehat{C}_{G O}(\sigma) \cong G L_{4}(q)=$ $C_{S O}(\sigma)(q \equiv-1(\bmod 4))$ и $\widehat{C}_{G O}(\sigma) \cong U_{4}(q)=C_{S O}(\sigma)(q \equiv-1(\bmod 4))$.

В случае инволюции II типа $\exists u \in \widehat{C}_{G O}(\sigma): u \sigma u^{-1}=-\sigma$, т.е. при переходе от $C_{G O}$ к $C_{\Omega}$ надо учитьвать удвоение централизатора. Если $\widehat{C}_{G O}(\sigma)=C_{S O}(\sigma)$, то инволюций II типа имеется по два класса. Порядки централизаторов инволюций II типа совпадают с порядком централизатора инволюции I типа при $\operatorname{dim} V^{+}=6, \operatorname{dim} V^{-}=2$ (инволюция $(6,2))$.

ЗАМЕчАНИЕ 2 . При необходимости $\sigma$ можно заменить на $-\sigma$, что позволяет считать $\operatorname{dim} V^{+}>\operatorname{dim} V^{-}$.

Эти три класса инволюций переставляются автоморфизмом "тройственности".

Система корней группы $O_{8}^{+}(q)=D_{4}(q)$ представляется диаграммой Дынкина следующим образом:

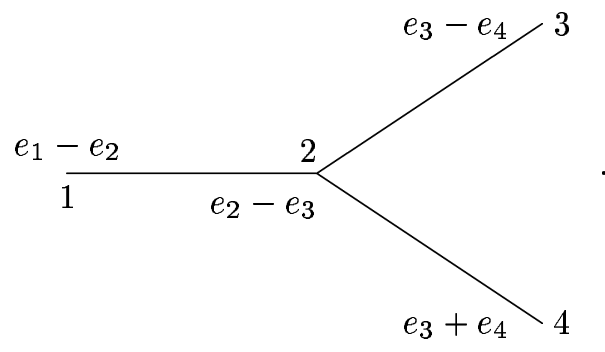

Центр группы Шевалле $D_{4}(q)$ состоит из картановских элементов

$$
h(t)=h_{1}\left(t_{1}\right) h_{2}\left(t_{2}\right) h_{3}\left(t_{3}\right) h_{4}\left(t_{4}\right)
$$


которые коммутируют с $x_{\alpha}$, а именно, для которых выполняется

$$
t_{i}^{2}=1, \quad t_{2}=1, \quad t_{1} t_{3} t_{4}=1
$$

$h_{2}(-1), h_{1}(a) h_{3}(a), h_{3}(a) h_{4}(a), h_{1}(a) h_{4}(a)$, где $a^{2}=-1,-$ представители четырех классов инволюций $(q \equiv 1(\bmod 4))$. При $q \equiv-1(\bmod 4)$ диагонализировать инволюции удается лишь расширением поля $F_{q}$ до $F_{q}^{2}$.

Инволюция $h_{2}(-1)$ соответствует инволюции I типа, где $\operatorname{dim} V^{+}=\operatorname{dim} V^{-}=4$ (инволюция $(4,4)$ ).

Допустим, что група $O_{8}^{+}(q)$ с автоморфизмом “тройственности” имеет нетривиальную $\varphi$-структуру.

Поскольку автоморфизм “тройственности” переставляет инволюции классов II типа и $(6,2)$, то можно считать, что в $T$ есть инволюция I типа.

Отвергнем наличие в $T$ инволюции $(6,2)$.

Поскольку $\sigma \in T$, то $\varphi(\sigma)=\sigma$. Допустим, что $\sigma$ - инволюция $(6,2)$.

Диагональная и полевая части автоморфизма $\varphi$ оставляют $\sigma$ инвариантным. Внутренняя часть переводит $\sigma$ в сопряженную инволюцию, а автоморфизм “тройственности" - в другой класс. Противоречие.

Поэтому $T$ содержит инволюцию $(4,4)$.

Централизатор этой инволюции в универсальной группе есть

$$
S L_{2}(q) \times S L_{2}(q) \times S L_{2}(q) \times S L_{2}(q)
$$

Множители порождаются корневыми централизаторами в группе $O_{8}^{+}(q)$. Надо расширить централизатор с помощью вейлевских элементов, переводящих $h_{2}(-1)$ в $h_{2}(-1) c$, где $c$ - элемент из центра универсальной групшы, а затем профакторизовать по последнему. Отметим, что централизатор в универсальной групе $\varphi$-инвариантен.

Рассуждения о составляющих $\varphi$, проведенные вьше, дают внутреннюю часть, совпадающую с сопряжением элементом из централизатора. Составляюшие $\varphi$, кроме графовой части, переводят в себя множители $S L_{2}(q)$. Графовая часть переставляет три из них. Поэтому $\varphi$ индуцирует нетривиальный автоморфизм на полупростом (при $q>3$ ) сечении с множителями типа $L_{2}(q)$. Но это давало бы нетривиальную $\varphi$-структуру на группе $L_{2}(q)$. Чтобы $\varphi$-структура на сечении была тривиальна, необходимо компенсировать “тройственность" внутренней частью, т.е. сопряжениями вейлевскими элементами. "Тройственность" переставляет корни в схеме Дьнкина следующим образом: $e_{1}-e_{2} \rightarrow e_{3}-e_{4}, e_{3}-e_{4} \rightarrow e_{3}+e_{4}$. Это дает: $e_{3} \rightarrow 0.5\left(e_{1}-e_{2}+e_{3}+e_{4}\right)$, $e_{4} \leftarrow 0.5\left(-e_{1}+e_{2}+e_{3}-e_{4}\right)$, а векторы 0.5 $\left(e_{1}-e_{2}+e_{3}+e_{4}\right), 0.5\left(-e_{1}+e_{2}+e_{3}-e_{4}\right)$ не входят в систему корней $\Sigma$ группы Вейля. Следовательно, при $q>3$ компенсации нет и нет графовой части в $\varphi$. Но этот случай уже разобран. Остается рассмотреть вариант при $q=3$, где группа $L_{2}(q)$ разрешима.

В этом случае "тройственность" дает порядок $\varphi$, делящийся на 3 . Поскольку Out = Aut / Inner $=\Sigma_{4}$, а элемент в $\Sigma_{4}$, чей порядок делится на 3 , есть в точности элемент порядка 3 , то $\varphi^{3}=1$ - внутренний автоморфизм, т.е. $\varphi^{3}(x)=s x s^{-1}$, где $s \in O_{8}^{+}(3)$.

Имеем $T \subset\left\{x: \varphi^{3}(x)=x\right\}=C(s) \subset C\left(s^{\alpha}\right)=C\left(s_{1}\right) \subset N\left\langle s_{1}\right\rangle$. В качестве $s_{1}$ можно взять элемент простого порядка. По $C$ - $N$-конструкции никакое $s$ не может быть сопряжено со своей степенью. По атласу [9] при нечетном порядке этого нет. Поэтому 
порядок $s$ должен быть четным, т.е. $s$ - полупростой элемент. А как отмечалось в п. 4.1, полупростой элемент сопряжен со своим обратным. Следовательно, элемент $s$ должен быть инволюцией для избежания противоречия.

На централизаторе $C(\sigma) \varphi^{3}=1$. Поэтому на квазигрупе $C(\sigma) / T L_{a}^{3}=1$, порядок квазигруппы $(C(\sigma): T) \equiv 1(\bmod 3)$ и 3 -часть $C(\sigma)$ содержится в $T$.

Подействуем автоморфизмом $\varphi$ на централизатор инволюции $(4,4)$. Диагональная и полевая составляющие $\varphi$ переводят $C(\sigma)$ в себя, а композиция графового и полевого автоморфизма переставляет множители $L_{2}(3)$ централизатора. Но в $L_{2}(3)$ есть элемент порядка 3. Поэтому он не может быть $\varphi$-неподвижньм. Противоречие.

\section{СПИСОК ЦИТИРОВАННОЙ ЛИТЕРАТУРЫ}

[1] Галкин В. М. Леводистрибутивные квазигруппы конечного порядка // Квазигруппы и лупы. Кишинев, 1979.

[2] Галкин В. М. О симметрических квазигруппах // УМН. 1984. Т. 39. №6.

[3] Галкин В. М. Леводистрибутивные квазигруппы. Дисс. ... д.ф..-м.н. М.: МГУ, 1991.

[4] Горенстейн Д. Конечные простые группы. М.: Мир, 1985.

[5] Дьедонне ЖК. Геометрия классических групп. М.: Мир, 1974.

[6] Стейнберг Р. Лекции о группах Шевалле. М.: Мир, 1975.

[7] Семинар по алгебраическим группам. М.: Мир, 1973.

[8] Thompson J. G. Finite groups with fixed-point-free automorphisms of prime order // Proc. Nat. Amer. Sci. USA. 1959.

[9] Conway J. H., Curtis R. T., Norton S. P. Atlas of Finite Groups. Oxford, 1985.

[10] Суворова О.В. $\varphi$-структура на лиевских конечных группах. Дисс. ... к.ф..-м.н. СанктПетербург: С-ПбГУ, 1998.

[11] Артин Э. Геометрическая алгебра. М.: Наука, 1969.

Нижегородский государственный технический университет 